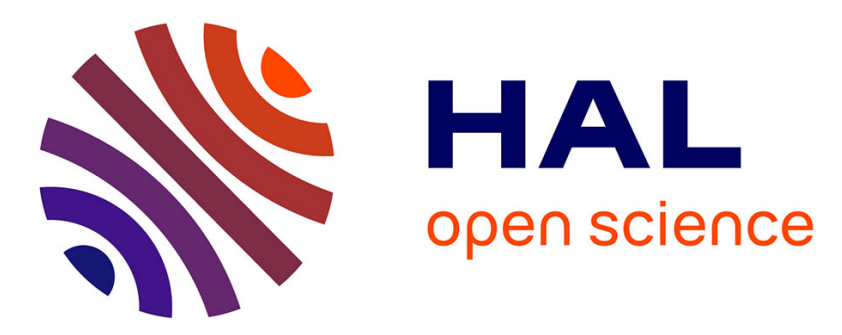

\title{
Extending Input Space of Tangible Dials and Sliders for Uncertain Input
}

\author{
Miriam Greis, Hyunyoung Kim, Andreas Korge, Céline Coutrix, Albrecht \\ Schmidt
}

\section{- To cite this version:}

Miriam Greis, Hyunyoung Kim, Andreas Korge, Céline Coutrix, Albrecht Schmidt. Extending Input Space of Tangible Dials and Sliders for Uncertain Input. Proceedings of the Thirteenth International Conference on Tangible, Embedded, and Embodied Interaction, Mar 2019, Tempe, France. ACM Press, pp.189-196, 10.1145/3294109.3300985 . hal-02413724

\section{HAL Id: hal-02413724 \\ https://hal.science/hal-02413724}

Submitted on 16 Dec 2019

HAL is a multi-disciplinary open access archive for the deposit and dissemination of scientific research documents, whether they are published or not. The documents may come from teaching and research institutions in France or abroad, or from public or private research centers.
L'archive ouverte pluridisciplinaire HAL, est destinée au dépôt et à la diffusion de documents scientifiques de niveau recherche, publiés ou non, émanant des établissements d'enseignement et de recherche français ou étrangers, des laboratoires publics ou privés. 


\section{Extending Input Space of Tangible Dials and Sliders for Uncertain Input}

\section{Miriam Greis}

University of Stuttgart

Germany

miriam.greis@codecentric.de

\section{Hyunyoung Kim}

Université Grenoble Alpes

CNRS, LIG

France

University of Stuttgart

Germany

hyunyoung.kim@imag.fr

\section{Céline Coutrix}

Université Grenoble Alpes

CNRS, LIG

France

University of Stuttgart

Germany

celine.coutrix@imag.fr

\section{Albrecht Schmidt}

University of Stuttgart

Germany

albrecht.schmidt@um.ifi.Imu.de

\section{Andreas Korge}

University of Stuttgart

Germany

andi-korge@web.de

Paste the appropriate copyright/license statement here. ACM now supports three different publication options: Permission to make digital or hard copies of part or all of this work for personal or classroom use is granted without fee provided that copies are not made or distributed for grofit or commercies full cion the first pan this work must be honored. For all other uses, contact the Owner/Author.

TEI '19, March 17-20, 2019, Tempe, AZ, USA

(C) 2019 Copyright is held by the owner/author(s)

ACM ISBN 978-1-4503-6196-5/19/03.

https://doi.org/10.1145/3294109.3300985

\begin{abstract}
Uncertainty is common when working with data and becomes more important as processing big data gains attention. However, no standard tangible interface element exists for inputting uncertain data. In this article, we extend the input space of two traditional TUIs: dial and slider. We present five designs that are based on dials and sliders and support uncertain input. We conduct focus group interviews to evaluate the designs. The interviews allow us to extend existing design requirements for parameter control UIs to support uncertain input.
\end{abstract}

\section{Author Keywords}

Tangible User Interfaces; Uncertainty; Input; Dial; Slider

\section{CCS Concepts}

Human-centered computing Haptic devices

\section{Introduction}

Users face uncertainty every day when they produce data themselves, e.g., recording calorie intake or booking flights. Whether users are the source of uncertainty or the mediator of uncertain data, input mechanisms need to support uncertain input to produce valid data containing uncertainty [1][5]. When the input uncertainty is well communicated, one can make better decisions when later analyzing the data [9][16]. 


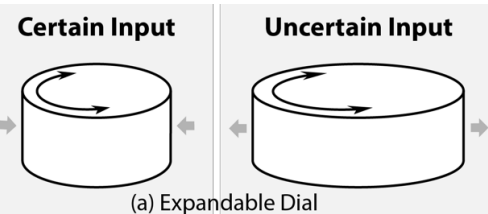

(a) Expandable Dial

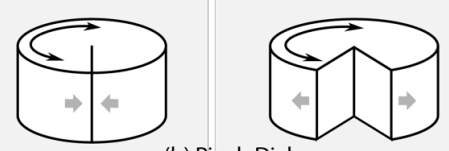

(b) Pinch Dial

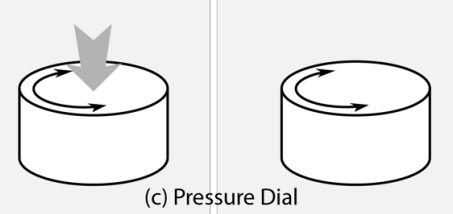

$\overleftrightarrow{1}$

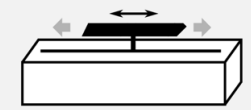

(d) Expandable Slider

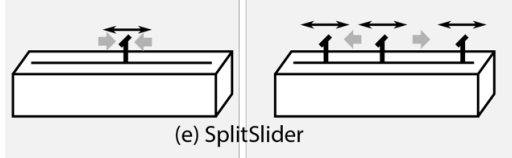

Figure 1. The extended input space of dial- and slider-based interfaces, to enable value and uncertainty input. The usual interactions (turning a dial or sliding a slider cursor) change value ( $\approx$ mean of a desired input). The added modalities (i.e., increasing diameter/open space/pressure/cursor size, and splitting the cursor respectively) allow to input uncertainty $(\approx$ variance of the input).
Communicating uncertainty can also increase transparency and reliability of the data [13][16].

Previous work has focused on the graphical representation of uncertain data [3][8][13][16] and capturing such data with mouse interface [5]. It inspires us to investigate how we can use tangible interfaces to support uncertain input. Tangible interfaces allow precise interaction [10], which can minimize uncertainty introduced by input methods. They also enable multiple interactions at the same time [17], which can be used to input estimated values and amount of uncertainty on one device.

We are especially interested in extending the onedimensional input space of dials and sliders to multidimensions, to allow input of both value and uncertainty on one device. Reconfigurable or shapechanging interfaces have demonstrated such capability [3][10][12], and we want to learn how the reconfigurability can support or hinder uncertain input.

In this work-in-progress, we explore the design of physical dials and sliders that can capture users' value and uncertainty on one device. We first use Morphees+ [11] features to design 5 controls that can input both value and uncertainty, which are based on ordinary dials and sliders. We then present low-fidelity prototypes and conduct a focus group study to gather feedback on them. Lastly, we derive and discuss preliminary design requirements for uncertain input.

\section{Background and Related Work}

Uncertainty and its Communication

Uncertainty, as described by Pang et al. [14], includes statistical variations or spread, errors and differences, minimum and maximum range values, noise, or missing data. Studies show that decision-making is better supported by capturing, modeling, and visualizing uncertain data [1][9][13][16]. For instance, communicating uncertainty can increase transparency and reliability of weather forecasts [13][16].

Pang et al. [14] also name three steps of data processing, in which uncertainty can be introduced: acquisition, transformation, and visualization. In data acquisition, uncertainty is inevitable due to inexact measurements. During transformation, the original data can be altered by a human or an algorithm. Lastly, visualization may introduce uncertainty as it does not usually use the same media as the original data.

In this paper, we focus on improving ways of capturing uncertainty during the transformation step. Research in capturing and reducing uncertainty has been largely focused on GUIs. Greis et al. [5] propose graphical probability distribution sliders with varying degrees of freedom. The study shows that users with limited statistical knowledge perform best using the range slider, which has two degrees of freedom. Users with more statistical knowledge perform better with sliders with three or more degrees of freedom.

Communicating Input Uncertainty with TUIs Even though TUIs have not explicitly addressed the problem of inputting uncertain data, several previous works could be used for this purpose.

Coutrix et al. suggest a resizable tangible slider [3] to compromise between travel length of the thumb and input accuracy. It could be also used to input uncertain data by interpreting the size of the slider as the 


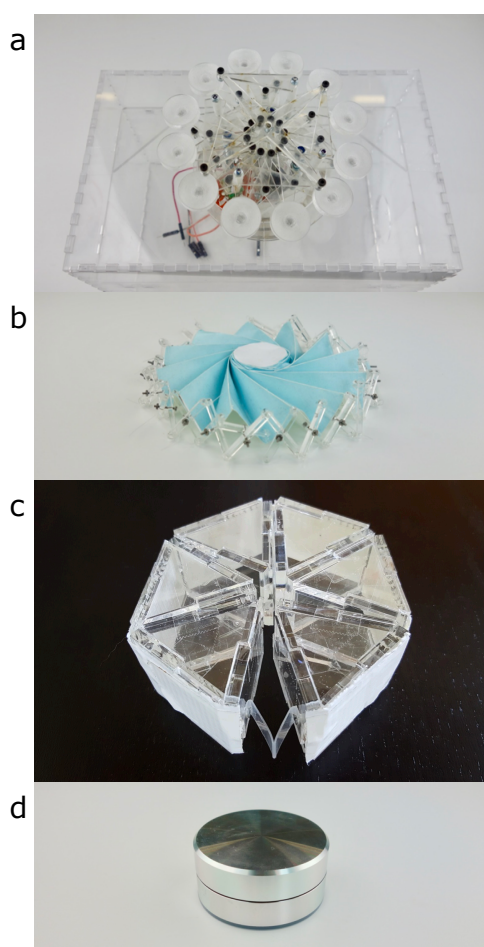

e
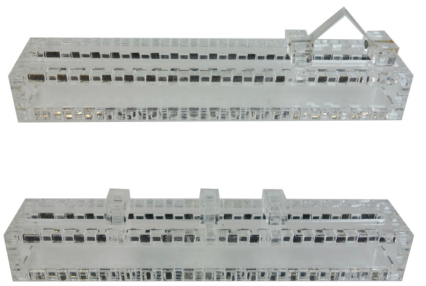

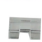

$=$

Figure 2. Low-fidelity prototypes for the explored designs for uncertain input dials/sliders. a,b) Expandable Dial, c) Pinch Dial, d) Pressure Dial, e) Expandable Slider, f) Split Slider. certainty. E.g., smaller size means less precision and thus more uncertainty.

The Inflatable mouse [12] could be also used for uncertain input. The mouse has an elastic chamber, and users can squeeze it to zoom a map in/out. For uncertain input, the device can input 2D data (e.g., $X-Y$ coordinate values with mouse pointing) with $1 \mathrm{D}$ uncertainty (squeezing).

\section{Design Exploration for Uncertain Input TUIs}

We first generated variations of conventiona continuous interfaces, dials and sliders. We used the existing interaction of rotating and sliding the devices for value input and added other modalities to input uncertainty. We used the features of the Morphees+ taxonomy [11] to design the additional modalities (see Figure 1). The designs quantify uncertainty in two ways: the dials and Expandable Slider (Figure 1a,b,c,d) can input an amount of uncertainty (i.e., 0-100\%), and SplitSlider (Figure 1e) can input a range of estimated values. When the device was for inputting the amount of uncertainty, we mapped the larger surface or open space with more uncertainty and less pressure with more uncertainty.

\section{Dial-Based Designs}

- Expandable Dial (Figure 1a) was driven by

Morphees+'s Area feature, which describes changes in area size on a surface. The dial's diameter can be increased or reduced, resulting in the area change at the top of the dial.

- Pinch Dial (Figure 1b) includes an open space that can be closed or opened, which was inspired by the Closure feature of Morphees+. The feature describes how much boundaries of a surface are "open".
- Pressure Dial (Figure 1c) can be pressed downwards in addition to its rotation, like Button+ [18]. It was driven by the Strength feature, the force needed to move a control point to another position.

\section{Slider-Based Designs}

- Expandable Slider (Figure 1d) was inspired by the Area feature similarly to Expandable Dial. It has a thumb that can be expanded to communicate uncertainty. The center of the thumb represents the medium of estimated values.

- SplitSlider (Figure 1e) was driven by Morphees+'s Modularity, which describes an object's ability to be split into multiple pieces. The design represents a standard slider in one-thumb mode. The thumb can be split to two or three thumbs to enter the range of estimated values or probability distribution (e.g., standard distribution) or estimated values.

\section{Low-Fidelity Prototype Implementation}

We implemented low-fidelity prototypes of all the design explorations, except for the pressure dial, where we used a consumer product [15] (Figure 2). We had six prototypes as we had two different prototypes for the Expandable Dial, one that could be stretched (Figure 2a) and one that could be squeezed (Figure $2 b)$. Here we briefly describe how they are implemented, and how they work.

Expandable Dial: Stretching Design. (Figure 2a) We use a Hoberman mechanism [7] to create an expandable circular shape. The center of the mechanism is connected to a rotational axis for value input. At the external corners, there are concave disks where users put their fingers on. Users can slightly 
press them and stretch the fingers inwards or outwards for inputting uncertainty.

Expandable Dial: Squeezing Design. (Figure 2b) We use to an aerospace design [6] to fold a paper sheet into a condensed shape. The tension of the paper keeps the dial expanded, and users squeeze it to change the diameter.

Pinch Dial. (Figure 2c) We create six equilateral prisms from a laser cutter and connect them into a hexagon, roughly resembling the round shape of a dial. They are held together with adhesive tape on the outer faces. We place a bent piece of plastic sheet between two prisms to act as a spring to open the dial. Users can close the dial by pinching the device.

Pressure Dial. (Figure 2d) We used a PowerMate Bluetooth [15] for the design. It has a spring inside to push the upper rotational part back when pressed. The device has only two states of height - pressed and notpressed.

Expandable Slider. (Figure 2e) We place a bent piece of plastic sheet between two thumbs of a laser-cut slider. The plastic sheet forced the thumbs to separate. Users can squeeze the thumbs for more certain input.

Split Slider. (Figure 2f) There are three thumbs on a laser-cut slider. The thumbs have grooves on them and users can place a U-shaped plastic piece to combine two thumbs together. Hence, the number of thumbs can be changed between one, two, and three.

\section{Focus Group Study}

We obtained general opinions on the input of uncertain data through focus group interviews. The interviews consist of two tasks: 1 ) understanding the causes of uncertain input and 2) evaluating our design

explorations. For the second task, we provided lowfidelity prototypes for the explored designs. We had two participant groups, and each group consisted of six people. Each focus group interview lasted about 50 minutes. We took pictures and recorded the audio during the interviews.

\section{Participants}

We recruited twelve participants (10 male, 2 female) between 20 and 34 years $(M=24.92, S D=3.65)$ via personal invitations. Their background varied, including social sciences and natural sciences. The participation was voluntary.

Task 1: Understanding the Causes of Uncertain Input (20min)

After the participants signed the consent form and provided their demographic information, they introduced themselves to the others. They then got sticky notes to write down answers for the following question: "Which scenarios can you think of where you were or could be uncertain about an input?"

\section{Results}

Participants found 20 scenarios where uncertain data input is needed. We report participants' scenarios that require them to communicate the uncertainty on continuous data (e.g., price), i.e., where this paper's interest is. We roughly grouped them into three categories: 
Lack of knowledge. Many scenarios implied knowledge that has never been learned in the first place. Participants mentioned complicated forms such as patient forms demanded by doctors, and these forms require rather uncommon knowledge. Another example was the alarm setting for e.g., cooking time, which might vary among kitchen equipment.

Loose constraints. Participants mentioned scenarios where uncertain input was caused by loose constraints. For instance, flight search was mentioned related to vacation planning. Here uncertainty could occur due to the fact that one might not have a specific price or traveling time in mind. For these data, the participants wanted input mechanisms allowing a range of alternatives. Participants also named portion input on cooking websites (every person might have their own understanding of "one portion").

Uncertain memories. Many scenarios can be traced back to missing or imprecise memories. This includes rarely used personal data, for which the participants mentioned their body size and weight.

Task 2: Evaluating the Designs (30 min) At the focus group interviews, we introduced our designs to the participants. Participants were grouped in three pairs. Each pair was randomly given three of the low-fidelity prototypes. They were asked to think about advantages, disadvantages, improvements or suitability of their respective prototypes for a given scenario among the scenarios found in the first task, and then each pair presented their findings. We repeated this step with the rest of the prototypes.
Results

Using thematic analysis [2], we identified preliminary requirements for designing tangible devices for uncertain input. The requirements are well-aligned with the requirements for the traditional rotational and linear input derived by Kim et al. [10]. It shows that the general purposes of the input interfaces are similar. However, the extended input space of the uncertain input TUIs also introduced new requirements that did not exist for traditional dials and sliders. There are seven requirements, which we grouped into four categories.

\section{FAST MANIPULATION.}

R1 Simultaneous input. In general, the participants liked to have simultaneous input of value and uncertainty. For instance, they complained that it was hard to press down the Pressure Dial while rotating because the resistance of the spring was too high. They also complained that the Pinch Dial was difficult to pinch when the opening is no longer between the thumbs and index fingers.

\section{PRECISE MANIPULATION.}

R2 Small intervals. Dials were preferred for small intervals in value input because they could have infinite input range. On the other hand, the input ranges of the sliders were limited by the slider sizes, hence their intervals for value input were larger than the dials'.

R3 No interference between inputs. The participants preferred not to have interference between the value and uncertainty inputs. For instance, when participants changed uncertainty (stretching or squeezing) with Expandable Dial, it could rotate the device by accident. When users grasp Pinch Dial, it applied some pressure on it, and it could cause unwanted change on uncertainty. These problems would be the same over all the devices except Split 
Slider since the inputs for value and uncertainty were separated.

R4 Easy finalization. The participants needed easy finalization of inputs. With all designs, users needed another input interface (e.g., button) to finalize their value and uncertainty input. With Expandable Dial or Split Slider, the participants were able to take off their hands from and then finalize their inputs. However, the springs on Pinch Dial and Expandable Slider forced them to hold the devices while finalizing inputs.

\section{OBSERVABLE UNCERTAINTY.}

R5 Visual feedback. The participants liked that Split Slider's thumb intervals give clear visual feedback on the uncertainty. In general, all the dials did not have clear visual feedback on how much uncertainty was expressed (e.g., whether Pinch Dial was 50\% open or $60 \%$ open).

R6 Force feedback. Participants also liked that Pinch Dial and Expandable Slider give force feedback on uncertainty. They mentioned that using pressure for certainty feels intuitive. However, it was unclear to them how much pressure represents which amount of uncertainty. This requirement seems mutually exclusive with the easy finalization since the springs can give force feedback and also cause unwanted movement of the openings/thumbs. Also, even though Pressure Dial gave force feedback, the upper button's travel distance was too short. Hence, the participants could not feel the force feedback was proportional to the travel distance.

SUPPORTING STATISTICAL KNOWLEDGE.

R7 Supporting both experts and novices. The participants liked that SplitSlider can express statistical knowledge and support both expert and novice users. The other designs could only express the amount of uncertainty without statistical distribution.

\section{Conclusion and Future Work}

In this article, we contributed to communicating input uncertainty by extending the input space of traditional TUIs. We believe our work bridge the field of uncertainty and tangible interfaces, can open many interesting research questions. We explored the design space and implemented low-fidelity prototypes. Lastly, we derived design requirements for uncertain input TUIs. For future work, we are interested in developing a working prototype and evaluating its ability to support more precise uncertain input, comparing with alternative input methods. We also wish to evaluate and refine the design requirements and investigate how to resolve conflicting design requirements (i.e., force feedback and easy finalization).

\section{Acknowledgment}

This work was supported by the French National Research Agency (ANR-11-LABX-0025-01, ANR-11EQPX-0002, ANR-15-CE23-0011-01) and the Alexander von Humboldt Foundation.

\section{References}

[1] Nadia Boukhelifa and David John Duke. 2009. Uncertainty Visualization: Why Might It Fail?. In CHI '09 Extended Abstracts on Human Factors in Computing Systems (CHI EA '09). ACM, New York, NY, USA, 4051-4056. DOI:

http://dx.doi.org/10.1145/1520340.1520616

[2] Virginia Braun and Victoria Clarke. 2006. Using thematic analysis in psychology. Qualitative Research in Psychology 3, 2 (Jan. 2006), 77-101. DOI:

http://dx.doi.org/10.1191/1478088706qp063oa

[3] Michael Correll and Michael Gleicher. 2014. Error Bars Considered Harmful: Exploring Alternate Encodings for Mean and Error. Visualization and 
Computer Graphics, IEEE Transactions on 20, 12 (2014), 2142-2151. DOI:

http://dx.doi.org/10.1109/TVCG.2014.2346298

[4] Céline Coutrix and Cédric Masclet. 2015. ShapeChange for Zoomable TUIs: Opportunities and Limits of a Resizable Slider. Springer International Publishing, Cham, 349-366. DOI:

http://dx.doi.org/10.1007/978-3-319-22701-6_27

[5] Miriam Greis, Hendrik Schuff, Marius Kleiner, Niels Henze, and Albrecht Schmidt. 2017. Input

Controls for Entering Uncertain Data: Probability Distribution Sliders. Proc. ACM Hum.-Comput.

Interact. 1, 1, Article 3 (June 2017), 17 pages. DOI: http://dx.doi.org/10.1145/3095805

[6] Guest, S.D., and Pellegrino, S. (1992).

"Inextensional Wrapping of Flat Membranes." First International Conference on Structural

Morphology, Montpellier, R. Motro and T. Wester, eds., 7-11 September, 203-215.

[7] Hoberman Associates, Inc. Retrieved from July 24, 2018 from http://www.hoberman.com/index.html.

[8] Jessica Hullman, Paul Resnick, and Eytan Adar. 2015. Hypothetical Outcome Plots Outperform Error Bars and Violin Plots for Inferences about Reliability of Variable Ordering. PLOS ONE 10, 11 (2015).

[9] Susan L. Joslyn and Jared E. LeClerc. 2012. Uncertainty forecasts improve weather-related decisions and attenuate the effects of forecast error. Journal of Experimental Psychology: Applied 18,1 (2012), 126-140. DOI: http://dx.doi.org/10.1037/a0025185

[10] Hyunyoung Kim, Céline Coutrix, and Anne Roudaut. 2018. KnobSlider: Design of a ShapeChanging UI for Parameter Control. In Proceedings of the $2018 \mathrm{CHI}$ Conference on Human Factors in Computing Systems (CHI '18). ACM, New York NY, USA, Paper 339, 13 pages. DOI: https://doi.org/10.1145/3173574.3173913
[11] Hyunyoung Kim, Celine Coutrix, and Anne Roudaut. 2018. Morphees+: Studying Everyday Reconfigurable Objects for the Design and Taxonomy of Reconfigurable UIs. In Proceedings of the $2018 \mathrm{CHI}$ Conference on Human Factors in Computing Systems (CHI '18). ACM, New York, NY, USA, Paper 619,14 pages. DOI: https://doi.org/10.1145/3173574.3174193

[12] Seoktae Kim, Hyunjung Kim, Boram Lee, Tek-Jin Nam, and Woohun Lee. 2008. Inflatable Mouse: Volume-adjustable Mouse with Air-pressuresensitive Input and Haptic Feedback. In Proceedings of the SIGCHI Conference on Human Factors in Computing Systems (CHI '08). ACM, New York, NY, USA, 211-224. DOI: http://dx.doi.org/10.1145/1357054.1357090

[13] Rebecca E. Morss, Julie L. Demuth, and Jeffrey K. Lazo. 2008. Communicating Uncertainty in Weather Forecasts: A Survey of the U.S. Public. Weather and Forecasting 23, 5 (2008), 974-991. DOI:

http://dx.doi.org/10.1175/2008WAF2007088.1

[14] Alex T. Pang, Craig M. Wittenbrink, and Suresh K. Lodha. 1997. Approaches to uncertainty visualization. The Visual Computer 13, 8 (1997), 370-390. DOI:

http://dx.doi.org/10.1007/s003710050111

[15] Powermate Bluetooth https://griffintechnology.com/us/powermatebluetooth

[16] Mark S. Roulston, Gary E. Bolton, Andrew N. Kleit and Addison L. Sears-Collins. 2006. A laboratory study of the benefits of including uncertainty information in weather forecasts. Weather and Forecasting 21, 1 (2006), 116-122. DOI: http://dx.doi.org/10.1175/WAF887.1

[17] Orit Shaer and Eva Hornecker. 2010. Tangible User Interfaces: Past, Present, and Future Directions. Found. Trends Hum.-Comput. Interact. 
3, 1\&\#8211;2 (Jan. 2010), 1-137. DOI: http://dx.doi.org/10.1561/1100000026

[18] Jihoon Suh, Wooshik Kim, and Andrea Bianchi. 2017. Button+: Supporting User and Context Aware Interaction through Shape-Changing
Interfaces. In Proceedings of the Eleventh International Conference on Tangible, Embedded, and Embodied Interaction (TEI '17). ACM, New York, NY, USA, 261-268. DOI:

https://doi.org/10.1145/3024969.3024980 